\title{
JBO LETTERS
}

\section{In vivo multiphoton nanosurgery on cortical neurons}

\author{
Leonardo Sacconi, ${ }^{\mathrm{a}, \mathrm{b}, \mathrm{t}, *}$ Rodney P. O'Connor, ${ }^{\mathrm{a},+}$ \\ Audrius Jasaitis, ${ }^{\mathrm{a}}$ Alessio Masi, ${ }^{\mathrm{a}, \mathrm{c}}$ Mario Buffelli, ${ }^{\mathrm{d}}$ and \\ Francesco S. Pavone ${ }^{\mathrm{a}, \mathrm{b}}$ \\ a University of Florence, European Laboratory for Nonlinear \\ Spectroscopy, Florence, Italy \\ bUniversity of Florence, Department of Physics, Florence, \\ Italy \\ ${ }^{\mathrm{C}}$ University of Florence, Department of Pathology and \\ Experimental Oncology, Florence, Italy \\ dUniversity of Verona, Department of Neurological and \\ Visual Sciences, Verona, Italy
}

\begin{abstract}
Two-photon microscopy has been used to perform high spatial resolution imaging of spine plasticity in the intact neocortex of living mice. Multiphoton absorption has also been used as a tool for the selective disruption of cellular structures in living cells and simple organisms. In this work, we exploit the spatial localization of multiphoton excitation to perform selective lesions on the neuronal processes of cortical neurons in living mice expressing fluorescent proteins. Neurons are irradiated with a focused, controlled dose of femtosecond laser energy delivered through cranial optical windows. The morphological consequences are then characterized with time lapse 3-D two-photon imaging over a period of minutes to days after the procedure. This methodology is applied to dissect single dendrites with submicrometric precision without causing any visible collateral damage to the surrounding neuronal structures. The spatial precision of this method is demonstrated by ablating individual dendritic spines, while sparing the adjacent spines and the structural integrity of the dendrite. The combination of multiphoton nanosurgery and in vivo imaging in mammals represents a promising tool for neurobiology and neuropharmacology research. @ 2007 Society of Photo-Optical Instrumentation Engineers. [DOI: 10.1117/1.2798723]
\end{abstract}

Keywords: in vivo imaging; long-term imaging; two-photon microscopy; laser ablation; laser surgery; neuroscience.

Paper 07177LR received May 17, 2007; revised manuscript received Aug. 7, 2007; accepted for publication Aug. 7, 2007; published online Oct. 22, 2007.

The nonlinear nature of two-photon fluorescence provides an absorption volume spatially confined to the focal region. The localization of the excitation is maintained even in strongly scattering tissues, allowing deep high-resolution microscopy. In combination with fluorescent protein expression techniques, two-photon microscopy has become an indispensable tool to image cortical plasticity in living mice. ${ }^{1}$ In parallel to its application in imaging, multiphoton absorption has also been used as a tool for the selective disruption of intracellular structures. ${ }^{2,3}$ A similar approach has also been applied

\footnotetext{
*Tel.: 055-457-2476; E-mail: Sacconi@lens.unifi.it
}

${ }^{\dagger}$ These authors contributed equally. in vivo, where two-photon imaging and laser-induced lesions have been combined.,

Other groups have taken advantage of multiphoton absorption to ablate or dissect individual neurons. Multiphoton nanosurgery has been performed in worms to study axon regeneration ${ }^{6}$ and dissect the role of specific neurons in behavior. ${ }^{7}$ Nevertheless, the potential of this technique has not been fully explored in the mammalian central nervous system. We demonstrate a method for performing multiphoton nanosurgery in the central nervous system of mice, and discuss the widespread applications in neurobiology research.

We exploited the spatial localization and deep penetration of multiphoton excitation to perform selective lesions on the neuronal processes of cortical neurons in mice expressing fluorescent proteins (thy1-YFP-H and thy1-GFP-M transgenic lines ${ }^{8}$ ). Neurons were irradiated with a focused, controlled dose of femtosecond laser energy delivered through a permanent cranial optical window. Transgenic mice aged 3 to 5 months were used in all experiments unless otherwise stated. Mice were deeply anaesthetized with an intraperitoneal injection of ketamine $(0.13 \mathrm{mg}$ per $\mathrm{g}$ body weight $)$ and xylazine $\left(0.01 \mathrm{mg} \cdot \mathrm{g}^{-1}\right)$. A craniotomy was performed and a $25-\mathrm{mm}^{2}$ region of the skull was removed, exposing the brain. An optical chamber was then constructed by covering the intact dura with physiological solution and a cover glass, and sealed with dental acrylic and cyanoacrylate glue. In experiments examining the specificity of the laser dissection, astrocytes were labeled with the red fluorescent dye sulforhodamine 101 (SR101) by a brief application of a 500-nM solution before placing the optical window. ${ }^{9}$ The morphological changes were then analyzed with time lapse 3-D two-photon imaging over a period of minutes to days after the procedure. The two-photon imaging and nanosurgery were performed using a custommade, upright, scanning microscope. A mode-locked Ti:sapphire Chameleon laser (Coherent Incorporated, Santa Clara, CA) provided the excitation light, which comprised 120-fs width pulses at a $90-\mathrm{MHz}$ repetition rate. The scanning head comprised two closed-loop feedback galvanometer mirrors VM500 (GSI Lumonics, Billerica, MA). The laser was focused onto the specimen by the objective lens XLUM $20 \times$, NA 0.95, WD 2 mm (Olympus, Tokyo, Japan). A closed-loop piezoelectric stage P-721 (Physik Instrumente, Karlsruhe/ Palmbach, Germany) allowed axial displacements of the objective up to $100 \mu \mathrm{m}$ with nanometric precision. The excitation wavelengths used were $935 \mathrm{~nm}$ (GFP-YFP) and $850 \mathrm{~nm}$ (sulforhodamine 101). The anaesthetized mouse was fixed onto the microscope stage using a miniaturized stereotaxic adapter (Stoelting, Wood Dale, IL). During imaging and nanosurgery, the body temperature was maintained at $37^{\circ} \mathrm{C}$ using a thermostatic blanket system. First, a $z$ stack was acquired with two-photon microscopy to obtain a 3-D reconstruction of the dendritic structure and select an $x, y$, and $z$ coordinate for our desired lesion site. Second, laser dissection was carried out by parking the laser beam at the chosen point and increasing the power from 30 to $170 \mathrm{~mW}$ (measured after the objective lens). The dose of laser energy was set by opening the shutter for $200 \mathrm{~ms}$. Finally, the laser power was decreased back to $30 \mathrm{~mW}$, and a 3-D image of the neuron was acquired to visualize the effect of laser irradiation. These experimental

1083-3668/2007/12(5)/050502/3/\$25.00 @ 2007 SPIE 


\section{JBO LETTERS}

a
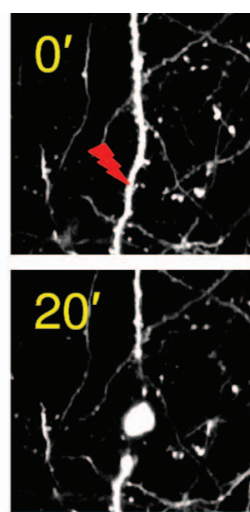

b
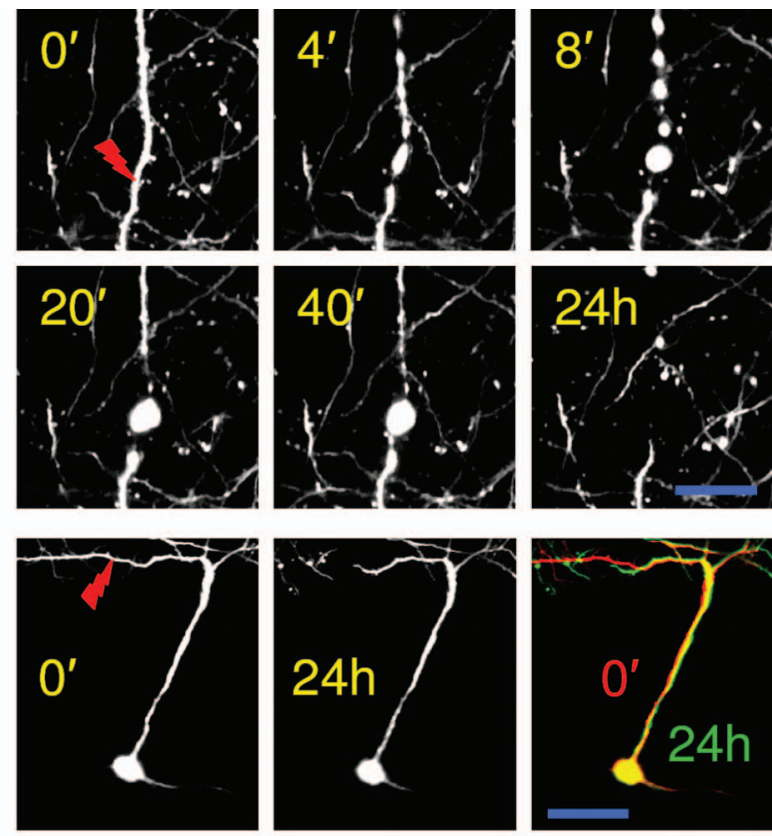

C
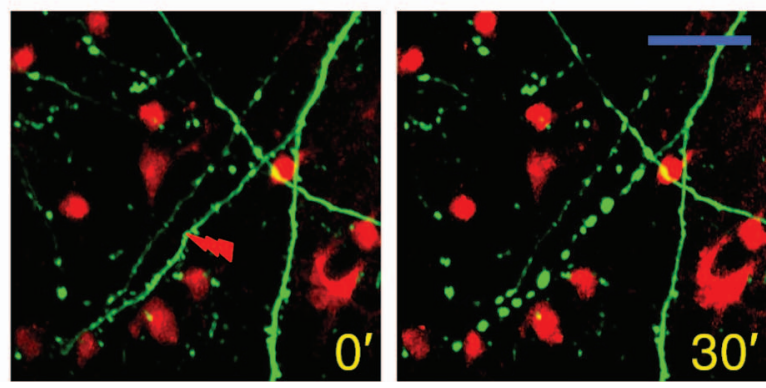

Fig. 1 Laser-induced lesion of a single dendrite. (a) Time-lapse images of an irradiated dendrite (at $\approx 100 \mu \mathrm{m}$ depth) in a GFP-M transgenic mouse. The dendrite was irradiated (as indicated by the tip of the red lightning) just after the acquisition of the first image. Each image is a maximum-intensity projection of a set of optical sections acquired at a $1-\mu \mathrm{m} z$ steps. Yellow numbers refer to the time from irradiation. Scale bar, $25 \mu \mathrm{m}$. (b) Maximum-intensity z projection (from 500 to $100 \mu \mathrm{m}$ depth) of a layer-5 pyramidal neuron before and $24 \mathrm{~h}$ after dendritic dissection (GFP-M line). The last panel shows an overlay of the neuron before (red) and after (green) laser dissection. This merge shows the integrity of the remaining structure after dissection. Scale bar, $60 \mu \mathrm{m}$. (c) The specificity of the laser dissection is demonstrated in two-color imaging experiments with labeled astrocytes. The laser nanosurgery is shown to disrupt only the GFP expressing dendrite (green), while the surrounding astrocytes (red) loaded with an intravital morphological dye (sulforhodamine 101) remain unaffected. Scale bar, $25 \mu \mathrm{m}$.

parameters were extrapolated from our previous investigations in cultured cells. ${ }^{3}$

In a first series of experiments, this methodology was applied to irradiate a single dendrite in the somatosensory cortex. Briefly, we found that the morphological consequences of irradiating a single dendrite can be grouped into two categories of biological response: 1. transient swelling with recovery, and 2. complete dendritic dissection. In the former case, we observed a swelling of the dendrite extending 5 to $25 \mu \mathrm{m}$ from the point of irradiation along the dendrite in both directions. The spines present on the irradiated dendrite temporarily disappeared with the swelling (data not shown), but returned after a period of minutes to hours with the original a
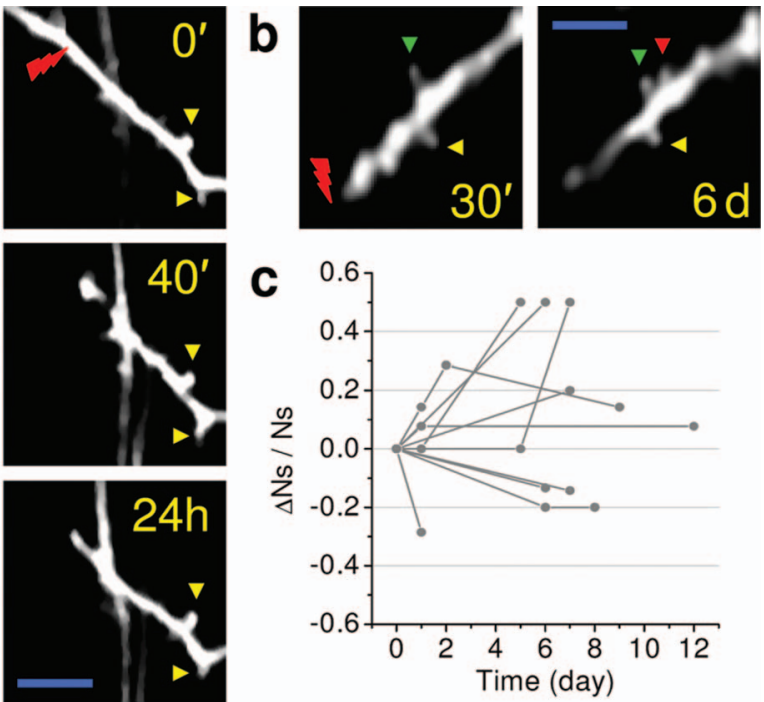

Fig. 2 Spine density following dendritic dissection. (a) Time-lapse images of an irradiated dendrite (at $\approx 200 \mu \mathrm{m}$ depth; YFP-H mouse). The arrowheads highlight the stability of the dendritic spines in the remaining part of the severed dendrite. Scale bar, $8 \mu \mathrm{m}$. (b) Long-term morphological changes of dendritic spines in an irradiated dendrite (at $\approx 150 \mu \mathrm{m}$ depth; YFP-H P30 mouse). Arrowheads: yellow, no change; green, morphological changes; red, new spine. Scale bar, $7 \mu \mathrm{m}$. (c) Percent variation of the number of spines (Ns) several days after laser dissection in 10 young mice (P30; YFP-H). The analysis was performed in the remaining portions $(10$ to $50 \mu \mathrm{m})$ of the irradiated dendrites.

a

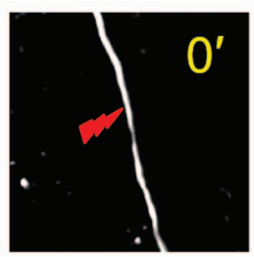

b
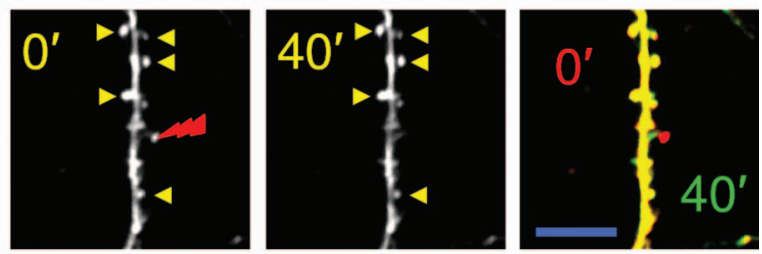

Fig. 3 Spatial resolution: single dendritic spine ablation. (a) Timelapse images of a dendrite (in GFP-M line) where, as indicated by the tip of the red lightning, the laser beam was parked at $\approx 0.5 \mu \mathrm{m}$ from the edge of the dendrite. This experiment was performed at $\approx 100 \mu \mathrm{m}$ depth. Scale bar, $25 \mu \mathrm{m}$. (b) Time-lapse images of a section of the dendrite where several spines are present (GFP-M). The single spine indicated by the red lightning symbol was irradiated just after the acquisition of the first image. The yellow arrowheads highlight the stability of the surrounding dendritic spines. The last panel shows an overlay of a pair of images from the sequence. The first image $(t$ $\left.=0^{\prime}\right)$ is shown in red and the last one $\left(t=40^{\prime}\right)$ in green. This panel emphasizes the spine stability and the absence of any swelling in the dendrite. Scale bar, $15 \mu \mathrm{m}$. 
shape of the dendrite. In the latter class of responses, the dendrite was completely severed. As shown in Fig. 1(a), after laser irradiation, the terminal end of the dendrite distal to the dissection point followed a sequence of swelling, degeneration, and disappearance. We can clearly observe [Fig. 1(b)] that the portion of dendrite no longer attached to the soma disappears. As shown in Fig. 1(a), the laser dissection can be performed with submicrometric precision and without any visible collateral damage to the surrounding neuronal structures. In addition, no damage or morphological changes were observed in astrocytes around the lesion site [see Fig. 1(c)]. The dendrite proximal to the dissection point and still connected to the soma regained its original shape and spine density [Fig. 2(a)]. Several days after dendritic dissection, we did not observe any changes in the remaining dendrite and surrounding area. On the other hand, we observed morphological changes in the dendritic spines on the remaining part of the severed dendrite [Fig. 2(b)]. As shown in Fig. 2(c), the number of spines [Ns in Fig. 2(c)] changed in the days following the nanosurgery. The continued expression of fluorescent proteins shows that the neuron is alive and, further, the remodeling of spines following the lesion demonstrated that the neuron is morphofunctionally active.

The underlying physical mechanisms of laser nanosurgery are not easily understood. In general, we must consider three potential mechanisms for the production of damage in the target structure: 1. thermal and chemical processes due to multiphoton absorption; 2. generation of large thermoelastic stresses; and 3. thermal, mechanical, and chemical processes emanating from optical breakdown (plasma formation) produced by a combination of multiphoton and cascade ionization processes. ${ }^{10}$

A full characterization of this nonlinear process is not feasible in vivo; therefore, we have focused our attention on providing practical guidelines for making use of this phenomenon. We found that the efficiency of laser dissection strongly depended on the level of fluorescent protein expression in the neuronal structure. Considering the maximum laser power available at the objective lens of our system $(\approx 170 \mathrm{~mW})$, we were able to perform laser-induced dendrite dissection at a maximum depth of 200 to $300 \mu \mathrm{m}$ in neurons with highly fluorescent dendrites. The spatial precision and the specificity of this method were demonstrated by irradiating an area very near the dendrite ( 1 to $0.5 \mu \mathrm{m}$ ) with no fluorescent features. As shown in Fig. 3(a), in this case we did not induce any visible alteration to the dendrite. This showed the high specificity and submicrometric precision of the laser dissection method. Commensurate with our previous findings, ${ }^{3}$ we were only able to ablate or dissect structures expressing fluorescent proteins. The spatial localization of multiphoton nanosurgery was maximally demonstrated by the ablation of individual dendritic spines [Fig. 3(b)]. As clearly shown in Fig. 3(b), we were able to remove a single dendritic spine without causing any visible collateral damage to the adjacent spines or parent dendrite.

The use of nonlinear optical methods in biology is continually undergoing developments and refinements. ${ }^{11}$ Two-photon microscopy is particularly useful in neuroscience where in vivo imaging has shown great potential in studying the structural correlates of learning and memory. ${ }^{12}$ The combination of multiphoton nanosurgery and in vivo imaging represents a promising tool for probing and disrupting neuronal circuits. The potential of using this precise optical method to perturb individual synapses cannot be overstated. Using multiphoton nanosurgery, the synaptic organization of the brain can now be teased apart in vivo to understand the microcircuitry of neuronal networks. We also envisage that this technique will provide insights in the mechanisms and dynamics of morphological remodeling in the brain following focal ischemic lesions. In addition, the response of the surrounding cells to this specific perturbation can be studied as a model of the reactive and degenerative changes in microstructure observed in certain neurodegenerative diseases. Finally, the combination of nanosurgery and in vivo imaging is an exciting tool for neuropharmacology research, because it can be used to evaluate the efficacy of systemic drugs on focal damage to the nervous system in vivo.

\section{Acknowledgments}

We thank G. Diana, A. Arcangeli, F. Keller, R. Panteri and D. Stampouli for assistance with the surgical procedure. We thank J. Sanes for providing the YFP-H and GFP-M mice. This research project has been supported by a Marie Curie Transfer of Knowledge Fellowship of the European Community's Sixth Framework Programme under contract number MTKD-CT-2004-BICAL-509761. Finally, we thank Ente Cassa di Risparmio di Firenze for contributing financial support to this project.

\section{References}

1. F. Helmchen and W. Denk, "Deep tissue two-photon microscopy," Nat. Methods 2(12), 932-940 (2005).

2. K. König, I. Riemann, P. Fischer, and K. J. Halbhuber, "Intracellular nanosurgery with near infrared femtosecond laser pulses," Cell Mol. Biol. (Paris) 45(2), 195-201 (1999).

3. L. Sacconi, I. M. Tolic-Norrelykke, R. Antolini, and F. S. Pavone, "Combined intracellular three-dimensional imaging and selective nanosurgery by a nonlinear microscope," J. Biomed. Opt. 10(1), 014002-014005 (2005).

4. J. A. Galbraith and M. Terasaki, "Controlled damage in thick specimens by multiphoton excitation," Mol. Biol. Cell 14(15), 1808-1817 (2003).

5. N. Nishimura, C. B. Schaffer, B. Friedman, P. S. Tsai, P. D. Lyden, and D. Kleinfeld, "Targeted insult to subsurface cortical blood vessels using ultrashort laser pulses: three models of stroke," Nat. Methods 3(2), 99-108 (2006).

6. M. F. Yanik, H. Cinar, H. N. Cinar, A. D. Chisholm, Y. Jin, and A. Ben-Yakar, "Neurosurgery: functional regeneration after laser axotomy," Nature (London) 432(7019), 822 (2004).

7. S. H. Chung, D. A. Clark, C. V. Gabel, E. Mazur, and A. D. Samuel, "The role of the AFD neuron in C. elegans thermotaxis analyzed using femtosecond laser ablation," BMC Neurosci. 7, 30 (2006).

8. G. Feng, R. H. Mellor, M. Bernstein, C. Keller-Peck, Q. T. Nguyen, M. Wallace, J. M. Nerbonne, J. W. Lichtman, and J. R. Sanes, "Imaging neuronal subsets in transgenic mice expressing multiple spectral variants of GFP," Neuron 28(1), 41-51 (2000).

9. A. Nimmerjahn, F. Kirchhoff, J. Kerr, and F. Helmchen, "Sulforhodamine 101 as a specific marker of astroglia in the neocortex in vivo," Nat. Methods 1(1), 31-37 (2004).

10. A. Vogel, J. Noack, G. Hüttman, and G. Paltauf, "Mechanisms of femtosecond laser nanosurgery of cells and tissues," Appl. Phys. B 81(8), 1015-1047 (2005).

11. W. R. Zipfel, R. M. Williams, and W. W. Webb, "Nonlinear magic: multiphoton microscopy in the biosciences," Nat. Biotechnol. 21(11), 1369-1377 (2003).

12. J. T. Trachtenberg, B. E. Chen, G. W. Knott, G. Feng, J. R. Sanes, E. Welker, and K. Svoboda, "Long-term in vivo imaging of experiencedependent synaptic plasticity in adult cortex," Nature (London) 420(6917), 788-794 (2002). 